\title{
DETECTION OF COCCIDIAN PARASITES AND THEIR CLINICAL MANIFESTATION, TREATMENT AND PROPHYLAXIS IN HIV INFECTED PATIENTS IN TRIBHUVAN UNIVERSITY TEACHING HOSPITAL
}

\author{
Basnet A.*, Sherchan J.B.*, Rijal B.*, Sharma S.*, Khadga P.* \\ *Department of Microbiology and Department of Internal Medicine, Tribhuvan University Teaching Hospital, Institute of \\ Medicine, Maharajgunj, Kathmandu, Nepal.
}

\begin{abstract}
The objective was to know the prevalence of coccidian parasites, their clinical manifestation, treatment and prophylaxis in HIV infected patients in Tribhuvan University Teaching Hospital, Nepal.

A total of 300 stool samples from 128 (64.00\%) HIV patient without previous history of treatment with antiretroviral therapy (ART), 72 (36.00\%) under ART treatment and 100 HIV seronegative control samples were collected and examined by wet mount, Kinyoun modified Ziehl Neelsen staining, Sheather's sucrose flotation and modified formalin-ethyl acetate sedimentation methods.

The coccidian parasites were detected in 22 (11.0\%) of the 200 HIV infected patients, 18 (9.0\%) without ART and 4 (2.0\%) with ART undertaking patients. Those without ART had majority of 11 (8.5\%) Cryptosporidium spp and those with ART had equal percentage of $2(0.03 \%)$ Cryptosporidium spp and $2(0.03 \%)$ Cyclospora spp. The prevalence of coccidian parasites was significantly higher in patients with diarrhea $(20 / 22)$ than in those without diarrhea $(2 / 22)$ (P value $<0.05)$. The drug therapy indices of the antibiotic, Cotrimoxazole given for 30 days in combination with ART for treatment and/or prophylaxis for opportunistic infections showed that long term treatment was needed for the clearance of coccidian parasites. Among 8 Cyclospora identified, $7.6 \%$ cleared from stool anlaysis after $30^{\text {th }}$ days of treatment likewise $15.3 \%$ of Cryptosporidium cleared after $45^{\text {th }}$ days of treatment.
\end{abstract}

In conclusion, Cryptosporidium followed by Cyclospora appeared to be the predominant coccidian parasite associated with diarrhea among HIV patients. Clinicians are requested to query for coccidian parasites to evaluate diarrhea in HIV patients. Cotrimoxazole is the drug of choice in curing coccidian parasites. So, it should be given along with ART as a treatment and/ or prophylaxis that act against both opportunistic infections as well as coccidian parasites. However its side effects should be evaluated for its long term prophylaxis.

Key words: AIDS; Coccidian; Diarrhea; HIV; ART; OIs.

\section{INTRODUCTION}

Acquired Immunodeficiency Syndrome (AIDS) represents the most severe sequel of immunosuppression caused by human immunodeficiency virus (HIV) and is a constellation of diseases reflecting the late manifestation of HIV-infection. A profound loss of the immune function results from the depletion of CD4 positive T-helper (CD4 +TH) cells caused directly by the virus and indirectly by virus mechanisms. ${ }^{1}$

Gastrointestinal tract (GIT) infections are among the most frequent in HIV and AIDS patients. It had been suggested that 50 to $93 \%$ of all such patients will have marked GI symptoms during the course of their illness. ${ }^{2,3}$

Reports indicate that diarrhea occurs in 30-60\% of AIDS patients in developed countries and in about $90 \%$ of AIDS patients in developing countries ${ }^{4}$. Chronic watery diarrhea associated with severe loss of weight (slime diseases) is often the presenting illness of HIV infection. ${ }^{2,3}$

Protozoan parasites especially coccidian parasites like Cyclospora cayetanensis, Crytosporidium parvum and Isospora belli, other enteric parasites like Entamoeba histoytica and Giardia lamblia accounts for a significant agents for diarrhea in HIV/AIDS population ${ }^{5,6,7}$.

Author for Correspondence: Amul Basnet/ J.B. Sherchan, Department of Microbiology, Tribhuvan University, Institute of Medicine, Maharajgunj, Kathmandu, Nepal, E-mail:amulbas@hotmail.com. 
The intracellular coccidian parasites that cause self-limiting diarrhea in healthy hosts, travelers or animal handlers, may cause protracted, voluminous diarrhea, often leading to profound wasting and weight loss in HIV/AIDS patients. These parasites are unusual as asymptomatic carrier in HIV/ AIDS but fatality is not uncommon. ${ }^{89}$ Cyclosporiasis has been reported with increasing frequency in the United States, Latin America, Nepal, Peru and New Guinea ${ }^{10-12}$. Since the diarrhoeal illness due to parasitic aetiology among HIV patients is on the rise during recent times, the present study was undertaken to examine the prevalence of enteric coccidian parasites among HIV/AIDS patients.

ART was initially introduced in 1986 and the first drug was Zidovudine. Initially mono and dual therapies were used but the problem of resistance emerged. At present 3 or more anti retroviral drugs (ARV) are recommended worldwide for the treatment of people with HIV infection. The ARV should be taken regularly, lifelong and studies indicate that $>95 \%$ adherence necessary for optimal virological suppression. In Nepal historic launching of ART was done at Teku Hospital in $12^{\text {th }}$ February 2004, the ART guideline was developed in 2004, at TUTH Maharajgunj, it was launched in $12^{\text {th }}$ January 2006.The ART was started in HIV /AIDS, WHO Clinical stage 1 and 2 if CD 4 count $<200$ cells/ $\mathrm{mm}^{3}$. In stage 3 therapy was considered if CD4 $<350$ but initiated before CD4 fallen to < 200, in stage 4 irrespective of CD4 count ART was started. ${ }^{5}$ The antibiotic Cotrimoxazole was given along with ART both for treatment and prophylaxis of opportunistic infections.

The criteria for diarrhea categorization were according to WHO criteria which were on the basis of diarrheal duration. Those having diarrhea for less than 2 weeks were classified as Acute diarrhea, those for two to four weeks as Intermittent and those having more than four weeks of diarrhea as Chronic diarrhea.

\section{MATERIALS AND METHODS}

The clinical and epidemiologic data presented were based on a cohort study from $1^{\text {st }}$ May till $15^{\text {th }}$ November, 2007. The clinical records and wet, stained and concentrated fecal smears from a representative sample of 200 of HIV/AIDS confirmed cases and 100 HIV seronegative control group patients were collected from Tribhuvan University Teaching Hospital, referred from indoor, outdoor department of TUTH and from different Volunteer Counseling and Testing centers, rehabilitation and referral centers located in and across the country like Nava Kiran Rehabilitation Center, National Association of PLHA in Nepal, Maiti Nepal, Jhapa, Pokhara, Birjung, Doti and Dhangadhi district. All the participants were informed with the research objectives and taken a written and verbal consent before obtaining sample. The patients were classified and characterized on the basis of demographic data, interview questionnaire, clinical profile and those with and without ART treatment, diarrheal history and CD4 count were noted.

The laboratory investigation part of the study was carried out at the Health Research Laboratory of Institute of Medicine, Tribhuvan University and at the Parasitology laboratory of Microbiology Department of Tribhuvan University Teaching Hospital, Maharajgunj, Kathmandu, Nepal. Patients were advised and instructed to collect about 20 gms of stool specimens for three consecutive days in a leak proof container provided to them. The treatment, prophylactic efficacy of Cotrimoxazole was observed in the positive coccidian parasite cases by analyzing the stool samples at $4^{\text {th }}, 14^{\text {th }}$ and $30^{\text {th }}$ days interval.

The parasitic examination was done by microscopy of saline wet mount, iodine wet mount and modified Ziehl Neelsen stain (Kinyoun method) to observe for the presence of characteristics coccidian and other intestinal parasites. The mounted slides were examined under low power (10X) followed by high power (40X) and in case of acid-fast stained smears, by oil immersion at $100 \mathrm{X}$ objective. The parasites were identified by their morphology and staining characteristics. The stool specimens were concentrated by Sheather's sucrose flotation and modified formalin-ethyl acetate sedimentation methods.

Chi-square $\left(\mathrm{X}^{2}\right)$ test was used to evaluate apparent differences for significance with Yates correction. Results were considered significant if the $P$ value was less than 0.05 .

\section{RESULTS}

In a total of 200 stool samples of HIV-infected patients, according to Anti Retroviral (ARV) therapy status, 128 (64.0\%) were not undertaking Anti Retroviral Therapy (ART) designated herein as PreART and 72 (36.0\%) were taking ART designated as ART patients. The overall prevalence of parasitic infection was found to be 56 (28.0\%) in which 22 (39.29\%) were coccidian parasites and 34 (60.71\%) other enteric parasites.

In the total 200 patients, 108(54\%) were male and 92 (46\%) were female, Out of 31 parasites positive cases in Males, 12 (38.7\%) had coccidian parasites and 19 (61.3\%) other enteric parasites, similarly out of 25 Females, 10 (40.0\%) had coccidian parasites and 15 (60.0\%) other enteric parasites.

Among 56 parasites positive cases according to ARV status in Table 1, 35 (62.5\%) were found in PreART patients and 21 (37.5\%) in ART.

Table1: Coccidian and other parasites positivity among ARV status.

\begin{tabular}{|l|l|l|l|}
\hline ARV status & Total Parasites & Coccidian & Other parasites \\
\hline PreART & $35(62.5 \%)$ & $18(51.4 \%)$ & $17(48.6 \%)$ \\
\hline ART & $21(37.5 \%)$ & $4(19.1 \%)$ & $17(80.9 \%)$ \\
\hline
\end{tabular}

Among coccidian parasites in both PreART and ART patients the highest prevalence was Cryptosporidium parvum which was 11 (31.4\%) and 2 (9.5\%) respectively followed by Cyclospora and Isopora. In the 100 samples Control group both Cryptosporidium 1 (3.5\%) and Cyclospora 1 (3.5\%) were found out of 28 (28\%) parasites positivity cases as shown in Table 2. 
Table2: Distribution of Coccidian and other parasites among in PreART, ART and Control groups.

\begin{tabular}{|l|l|l|l|l|l|l|}
\hline \multirow{2}{*}{ Parasites detected } & \multicolumn{2}{|c|}{ PreART } & \multicolumn{2}{c|}{ ART } & \multicolumn{2}{c|}{ CONTROL } \\
\cline { 2 - 7 } & \multicolumn{1}{|c|}{ Frequency } & \multicolumn{1}{c|}{ Percentage } & Frequency & \multicolumn{1}{c|}{ Percentage } & \multicolumn{1}{c|}{ Frequency } & \multicolumn{1}{c|}{ Percentage } \\
\hline C. parvum & $11 / 35$ & $31.4 \%$ & $2 / 21$ & $9.5 \%$ & $1 / 28$ & $3.5 \%$ \\
\hline C. cayetanensis & $6 / 35$ & $17.2 \%$ & $2 / 21$ & $9.5 \%$ & $1 / 28$ & $3.5 \%$ \\
\hline I. belli & $1 / 35$ & $2.9 \%$ & $0 / 21$ & $0.0 \%$ & 0 & 0 \\
\hline G. lamblia & $8 / 35$ & $22.6 \%$ & $6 / 21$ & $28.6 \%$ & $6 / 28$ & $21.4 \%$ \\
\hline E. histoytica & $3 / 35$ & $8.6 \%$ & $3 / 21$ & $14.3 \%$ & $4 / 28$ & $14.2 \%$ \\
\hline A. duodenale & 0 & 0 & 0 & 0 & $7 / 28$ & $25.0 \%$ \\
\hline A. lumbricoides & $3 / 35$ & $8.6 \%$ & $4 / 21$ & $19.1 \%$ & $5 / 28$ & $17.8 \%$ \\
\hline T. trichuria & $2 / 35$ & $5.7 \%$ & $3 / 21$ & $14.3 \%$ & $3 / 28$ & $10.7 \%$ \\
\hline S. stercoralis & $1 / 35$ & $2.9 \%$ & $1 / 21$ & $4.8 \%$ & $1 / 28$ & $3.5 \%$ \\
\hline
\end{tabular}

Table3: Coccidian parasites and their association with diarrheal characteristics

\begin{tabular}{|l|l|l|l|l|l|}
\hline \multirow{2}{*}{ Coccidian Parasites } & \multirow{2}{*}{ Positive cases } & \multicolumn{3}{|c|}{ Diarrhea Types } & \multirow{2}{*}{ No Diarrhea } \\
\cline { 3 - 5 } & & \multicolumn{1}{|c|}{ Chronic } & \multicolumn{1}{|c|}{ Intermittent } & Acute & \\
\hline C. parvum & $13(6.5 \%)$ & $6(46.2 \%)$ & $3(23.1 \%)$ & $3(23.1 \%)$ & $1(7.7 \%)$ \\
\hline C. cayetanensis & $8(4.0 \%)$ & $3(37.5 \%)$ & $1(12.5 \%)$ & $3(37.5 \%)$ & $1(12.5 \%)$ \\
\hline I. belli & $1(0.5 \%)$ & $1(100.0 \%)$ & $0(0.0 \%)$ & $0(0.0 \%)$ & $0(0.0 \%)$ \\
\hline
\end{tabular}

The association of coccidian parasites to diarrhea types has shown highest prevalence of Cryptosporidium parvum in Chronic diarrhoea 6 (46.2\%) followed by Cyclospora cayetanensis which showed equal prevalence in both Chronic diarrhoea 3 (37.5\%) and Acute diarrhoea 3 (37.5\%) as shown in the Table 3.There is significant association of diarrhea between PreART and ART cases. (P value $=<0.05$ )

The drug therapy indices of the antibiotic - Cotrimoxazole (Trimethoprim/sulfamethoxazole: $160 / 800 \mathrm{mg}$ ) per oral two times for 30 days in case of 22 coccidian parasites positive cases in combination with ARV and followed up stool analysis at mean days of $4^{\text {th }}, 14$ th and $30^{\text {th }}$ days of treatment had revealed Cyclospora 7 cases cured in $4^{\text {th }}$ days, 1 case in $14^{\text {th }}$ days and 1 case recurred in $14^{\text {th }}$ days but cured after $30^{\text {th }}$ days. Similarly Cryptosporidium 7 cases cured in $4^{\text {th }}$ days, 6 cases cured in $14^{\text {th }}$ days and 2 cases recurred in $30^{\text {th }}$ days but cured after $45^{\text {th }}$ days of prophylaxis. Likewise Isospora belli 1 case cured after $14^{\text {th }}$ days of treatment.

The prevalence of coccidian parasites was highest in the HIV patients with CD4 count below 200 per cumm and lowest in those having CD4 count more than 350 per cumm of blood as shown in the figure 1.

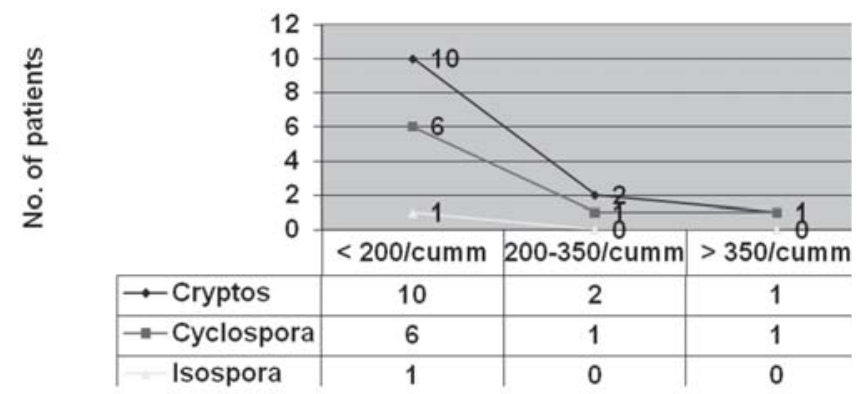

Fig.1: Distribution of different types of coccidian according to CD4 cell counts range.

The prevalence of coccidian parasites was higher in June and July comparable to other months as shown in the figure 2 .

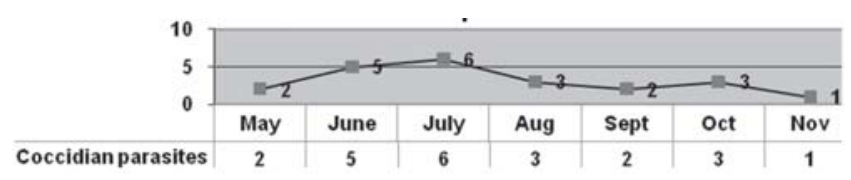

Fig.2: Distribution of coccidian parasites according to the months

\section{DISCUSSION}

Laboratory diagnosis of the coccidian parasites was based on the demonstration of oocysts in faeces by wet mount examination and modified Ziehl Neelsen stain (Kinyoun method). The oocysts showed distinct acid fast staining characteristics, pinkish in color and their sizes were remarkable. Both Sucrose floatation method and modified formalin-ethyl acetate sedimentation methods were equally valuable to detect oocysts of coccidian parasites.

Study had shown 56 (28.0\%) parasite positive in total 200 HIV-infected patients, 22 (11.0\%) were coccidian and 34 (17.0\%) other enteric parasites. Similarly in HIV seronegative control group coccidian were 2 (2.0\%) and other enteric parasites 26 (26.0\%). There was significant difference observed in the prevalence of coccidian parasites among HIVs and non HIVs group. In HIV group, the most predominant coccidian parasite was Cryptosporidium parvum 13(6.5\%) followed by Cyclospora cayetanensis 8(4.0\%) and Isospora belli $1(0.5 \%)$. Whereas Pape JW et al had reported an overall prevalence of Isospora belli was higher among three coccidian in HIV infection. ${ }^{16}$ and Rai et al had reported the prevalence of Cryptosporidium parvum 5.2\% in HIV+ subjects. ${ }^{14}$

Giardia lamblia 14(7.0\%) had shown the highest prevalence among protozoa and Ascaris lumbricoides 5(3\%) among helminthes. Rai et al had reported Entamoeba histoytica 3 (7.9\%) and Trichuris trichuria 19 (50.0\%) as highest prevalence among HIV patients. ${ }^{14}$

Sherchand et $\mathrm{al}^{10,12}$ has reported Cryptosporidium parvum as third most prevalent protozoa associated with diarrhea in Nepal with prevalence rates of $6.8 \%$ and $4.4 \%$ in 1996 and 
1997 respectively. This study had shown increased prevalence rate of $7.5 \%$ in case of HIV/AIDS patients. This might be due to increase susceptibility of these patients to the infection. ${ }^{13}$

Cryptosporidium parvum had shown higher prevalence in association with Chronic diarrhea 6 (46.2\%), also overall coccidian parasites were significantly higher in patients with diarrhea (20/22) than in those without diarrhea (2/22) (P value $<0.05$ ). Satheesh KS et al had found $39 \%$ of patients with diarrhea infected with coccidian parasites in 2002. ${ }^{15}$

The drug efficacy of Cotrimoxazole had shown the recurrence of Cryptosporidium 16\% and Cyclospora 33.3\% but had cleared after long term prophylaxis. Similar study conducted in Haiti by Jean William et al had shown recurrence of Cyclospora $42.8 \%$ in the mean days of $30^{18}$ and Pape JW et al. had observed similar recurrence of Isospora belli in his study. ${ }^{16}$

The distribution of coccidian parasites was higher in June and July compare to other months which was similar in study conducted by Sherchand et al. ${ }^{10,12,19}$

\section{CONCLUSION}

The routine examination of stool with special interest to coccidian parasites will help in the management of gastrointestinal infections in HIV-infected patients with or without diarrheal history.

The rate of coccidian parasites infection was found to be decreased in patients having ART compared to the PreART patients this may be due to the antibiotic Cotrimoxazole, which was given to all ART patients for treatment and/or prophylaxis of opportunistic infections. Cotrimoxazole is known to be the drug of choice for the coccidian parasites. Hence the Cotrimoxazole should be given to all PreART patients regardless of the time for start of ARV therapy. But the side effects of its long term usage should be monitored. The importance of other intestinal parasitic infections should not be neglected as they may be implicated in the cause of wasting disease of HIV to AIDS. This kind of study should be done in different geographical settings, ethnicity and age group to obtain precise data for interpretation of the exact status in this regard.

\section{ACKNOWLEDGEMENT}

I am deeply indebted to Prof. Dr. Bharat Mani Pokhrel, for his kind approval and support to undertake this thesis under the guidance of my supervisors. I am extremely grateful to my Principal Supervisor Prof. Dr. Jeevan Bahadur Sherchand and other supervisors; Assoc. Prof. Dr. Basista Rijal, Assoc. Prof. Dr Sashi Sharma and Assoc. Prof. Dr. Prem Khadga for their guidance and constant support. I would like to thank the team of Counselling Centre of TUTH, HIV committee member especially to Ms Uma Chitrakar and other members and directors of HIV and other venereal diseases referral centres for providing me the chance to collect my thesis samples. Similarly I would like to thank technical staff members of TUTH and Health Research Laboratory and Department of Microbiology, TUTH, Institute of Medicine.

\section{LITERATURE CITED}

1. Isenberg, H.D. 2004. Clinical Microbiology Procedures Handbook. 2nd edition. American Society for Microbiology, Washington, DC.

2. Scharschmidt and Fieldman. 1999. Protozoal infections in patients with AIDS. Cryptosporidiosis, isosporiasis, cyclosporiasis, and microsporidiosis. Infect Dis Clin North Am. 8(2): 483-98.

3. Fisseha. 1996. Gastrointestinal and hepatobiliary manifestations of human immunodeficiency virus infection. Trop Med Hyg. 84(1): 66-7.

4. Framm and Soave. 1997. Prevalence of intestinal parasitic infections in patients with acquired immunodeficiency syndrome in developing countries. Int J Infect Dis. 3(4): 203-6.

5. National Guideline on Anti- Retroviral (ARV) Therapy. 2005 (2062). Ministry of Health and Population. National Center for AIDS and STD control, Kathmandu, Nepal

6. Kang. 2000. Protozoal infections in patients with AIDS. Cryptosporidiosis, isosporiasis, cyclosporiasis, and microsporidiosis. Infect Dis Clin North Am. 8(2): 483-98.

7. Kava. 2002. Diagnosis and management of protozoan parasites in HIV/AIDS. Clin Infect Dis. 29(5): 1117-25.

8. Current. 1991. Cryptosporidiosis associated with ozonated apple cider. Emerg Infect Dis. 12(4): 684-6.

9. Kairon, R. 2003-2004. Study on opportunistic enteric parasites in HIV-seropositive adult patients hospitalized for diarrhea. National AIDS Research Institute. Annual Report.

10. Sherchand, J.B. and Cross, J.H. 2004. Cyclospora cayetanensis in Nepal. A study of Microbiological and Epidemiological aspects. NHRC.

11. Ortega, Y.R., Arrowood, M., Cyclospora and Isospora. 2003. Manual of Clinical Microbiology. 8th ed. P. 2008-16.

12. Sherchand, J.B., Cross, J.H. 2001. Emerging pathogen Cyclospora cayetanensis infection in Nepal. Southeast Asian J Trop Med Public Health. 32: 143-150.

13. Lawn, S.D. 2004. AIDS in Africa: the impact of coinfections on the pathogenesis of HIV-1 infection. J. Infect. Dis. 48(1): 1-12.

14. Rai, S.K. et al. 2006. Intestinal Parasitic Infections among HIV Seropositive and High Risk Group Subjects for HIV Infection in Nepal. Nepal Medical College Journal. 8(3): 166-170.

15. Satheesh, K.S. et al. 2002. Intestinal parasitic infections in HIV-infected patients with diarrhea in Chennai. Indian $J$ Med Microbiol. 20(2): 88-91.

16. Pape, J.W. et al. 1994. Cyclospora infection in adults infected with HIV. Annals Int Med. 121(9): 654-7.

17. Soave, R. 1988. Cryptosporidiosis and isosporiasis in patients with AIDS. Infect Dis Clin North Am. 2(2): 485-93.

18. Jean William et al. 2004. Cryptosporidiosis and other intestinal parasitic infections in patients with chronic diarrhea. Saudi Med J. 25(9): 1204-7. 
19. Sherchand, J.B. et al. 1999. Study of Cyclospora cayetanensis in health care facilities, sewage water, and green leafy vegetables in Nepal. Southeast Asian J Trop Med Public Health. 30: 58-63.

20. Nwokeidiuko, Ozumba. 2002. Diarrheal characteristics of AIDS patients in Nigeria. Ann Intern Med. 137: 381-433.

21. Guerrant, R.L. et al. 1990. Diarrhea in developed and developing countries: magnitude, special settings, and etiologies. Rev. Infect. Dis. 12 (Suppl 1): S41-S50.

22. Mohandas, K. et al. 2002. Prevalence of intestinal parasitic pathogens in HIV-seropositive individuals in Northern India. Jpn J Infect Dis. 55: 83-4.
23. United States Department of Health and Human Services. 2004. A Guide to Primary Care for People with HIV/AIDS. Retrieved on 2006-07-03.

24. Verdier, R.I. et al. 2000. Trimethoprim-sulfamethoxazole compared with ciprofloxacin for treatment and prophylaxis of Isospora belli and Cyclospora cayetanensis infection in HIVinfected patients. A randomized, controlled trial. Ann Intern Med. 32(11): 885-8.

25. Centers for Disease Control and Prevention. Cyclospora infection: information for health care providers. CDC Website. 